\title{
Influence of Channel Layer Thickness on the Electrical Performances of Inkjet-Printed In-Ga-Zn Oxide Thin-Film Transistors
}

\author{
Ye Wang, Xiao Wei Sun, Senior Member, IEEE, Gregory Kia Liang Goh, \\ Hilmi Volkan Demir, Member, IEEE, and Hong Yu Yu, Senior Member, IEEE
}

\begin{abstract}
Inkjet-printed In-Ga-Zn oxide (IGZO) thin-film transistors (TFTs) with bottom-gate bottom-contact device architecture are studied in this paper. The impact of the IGZO film thickness on the performance of TFTs is investigated. The threshold voltage, field-effect mobility, on and off drain current, and subthreshold swing are strongly affected by the thickness of the IGZO film. With the increase in film thickness, the threshold voltage shifted from positive to negative, which is related to the depletion layer formed by the oxygen absorbed on the surface. The field-effect mobility is affected by the film surface roughness, which is thickness dependent. Our results show that there is an optimum IGZO thickness, which ensures the best TFT electrical performance. The best result is from a 55-nm-thick IGZO TFT, which showed a field-effect mobility in the saturation region of $1.41 \mathrm{~cm}^{2} / \mathrm{V} \cdot \mathrm{s}$, a threshold voltage of $1 \mathrm{~V}$, a drain current on/off ratio of approximately $4.3 \times 10^{7}$, a subthreshold swing of $384 \mathrm{mV} / \mathrm{dec}$, and an off-current level lower than $1 \mathrm{pA}$.
\end{abstract}

Index Terms-Film thickness, In-Ga-Zn oxide (IGZO), inkjet printing, thin-film transistors (TFTs).

\section{INTRODUCTION}

$\mathbf{I}$ NKJET-PRINTED THIN-FILM TRANSISTOR (TFT) technology has received great attention as a low-cost alternative to conventional silicon-based technologies [1]. This technology is expected to result in many benefits. First, it is a low-waste and maskless process. Deposition and patterning are accomplished by ink jetting, reducing material usage and process complexity [2]. In addition, inkjet printing is amenable to roll-to-roll manufacturing fabrication on flexible substrate in

Manuscript received August 3, 2010; revised October 25, 2010; accepted October 26, 2010. Date of publication December 10, 2010; date of current version January 21, 2011. This work was supported in part by the Academic Research Fund (RGM 44/07) Ministry of Education, Singapore and Singapore and Science and Engineering Research Council Public Sector Fund (092 101 0057) of Agency for Science Technology and Research, Singapore. The review of this paper was arranged by Editor H. Jaouen.

Y. Wang and H. Y. Yu are with the School of Electrical and Electronic Engineering, Nanyang Technological University, Singapore 639798.

X. W. Sun is with the School of Electrical and Electronic Engineering, Nanyang Technological University, Singapore 639798 and the Department of Applied Physics, College of Science, Tianjin University, Tianjin 300072, China (e-mail: exwsun@ntu.edu.sg).

G. K. L. Goh is with the Institute of Materials Research and Engineering, Agency for Science, Technology and Research (A*STAR), Singapore 117602.

H. V. Demir is with the School of Electrical and Electronic Engineering and the School of Physical and Mathematical Sciences, Nanyang Technological University, Singapore 639798, and also with the Department of Electrical and Electronics Engineering and Department of Physics, Bilkent University, Ankara 06800, Turkey.

Color versions of one or more of the figures in this paper are available online at http://ieeexplore.iee.org.

Digital Object Identifier 10.1109/TED.2010.2091131 the ambient condition [3]. This renders more process flexibility, high overall thoughput, and ultralow cost. One of the critical materials for printed TFTs is a stable and jettable semiconductor solution. Research on solution-processable semiconductor materials is focused on silicon and organics [4]-[7].

Metal-oxide semiconductor is advantageous in terms of mobility and stability, and some groups have done plenty of work in this area, especially by inkjet printing method [8][10]. In-Ga-Zn oxide (IGZO) is a good alternative channel layer material for TFTs, compared to conventional a-Si and poly-Si [11]-[14]. IGZO is transparent in the visible region due to the large band gap and has a high mobility, even for an amorphous structure due to s-electron conduction [11]. High mobility is essential for current-driving devices such as organic light-emitting diodes and for building integrated electronics/drivers for system-on-glass. Up to now, most IGZO TFTs are fabricated by magnetron sputtering [13]-[17] and pulsed laser deposition [11]. To reduce the cost, IGZO TFTs have been fabricated by spin-coating [18]-[20]. Kim et al. reported staggered IGZO TFTs (bottom-gate top-contact) by inkjet printing [21]. However, the mobility was $0.03 \mathrm{~cm}^{2} / \mathrm{V} \cdot \mathrm{s}$, which was lower than that of the spin-coated IGZO TFTs. It is known that, compared with bottom-gate top-contact, bottomgate bottom-contact is more feasible to be used in the inkjetprinted TFT technology process. Therefore, in this paper, we applied inkjet printing method to fabricate IGZO TFTs with bottom-gate bottom-contact architectures for the first time. Here, we focus on investigating the impact of channel layer thickness on the electrical performance of IGZO TFT prepared by inkjet printing technology.

\section{EXPERIMENTAL DETAILS}

The IGZO ink was prepared by dissolving $0.1 \mathrm{M}$ of zinc acetate dehydrate $\left[\mathrm{Zn}(\mathrm{OAc})_{2} \cdot 2 \mathrm{H}_{2} \mathrm{O}\right], 0.1-\mathrm{M}$ indium chloride, and $0.0025-\mathrm{M}$ gallium chloride (the atom ratio of $\mathrm{Ga}: \mathrm{In}: \mathrm{Zn}=$ 25:100:100) in 2-methoxyethanol. A 0.2-M monoethanolamine (MEA) was then added in the precursor solution as a sol-gel stabilizer. After thoroughly mixing all components, the solution was stirred at $50{ }^{\circ} \mathrm{C}$ for $2 \mathrm{~h}$ and then aged for $24 \mathrm{~h}$. A heavily doped $p^{+}$-Si wafer (carrier concentration $\sim 10^{19} \mathrm{~cm}^{-3}$ ) was employed as the bottom gate of the TFT. A 150-nm-thick $\mathrm{SiO}_{2}$ film was thermally grown on top of the silicon wafer, which was used as the gate dielectric layer. A 150-nm-thick ITO film was deposited by dc sputtering as the source and drain electrodes 

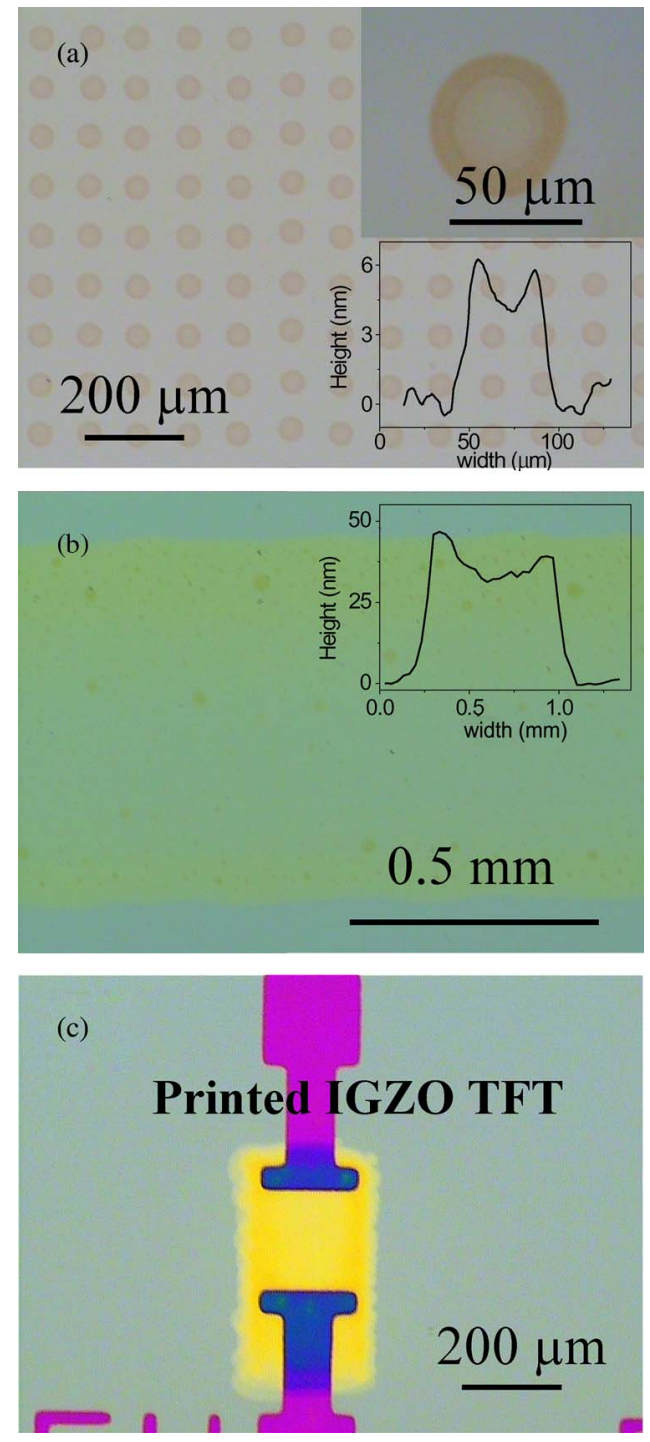

Fig. 1. (a) Inkjet-printed IGZO dots, (b) single line, and (c) IGZO TFT with bottom-gate bottom-contact architecture. Insets in (a) are the printed single dot and its 2-D profile. In addition, inset in (b) is the 2-D profile of the printed single line.

were patterned on the $\mathrm{SiO}_{2}$ surface by liftoff. In most cases, before IGZO printing, the silicon substrate with patterned ITO was cleaned by acetone, isopropanol, and de-ionized water sequentially, followed by nitrogen blow dry. The IGZO ink was deposited by a DMP 2831 inkjet printer. This process was repeated several times to obtain the desired film thickness on the prepared substrate and heated at $300{ }^{\circ} \mathrm{C}$ in the air by a hotplate for $10 \mathrm{~min}$. Postannealing was performed at $500{ }^{\circ} \mathrm{C}$ for $1 \mathrm{~h}$ in air by a common laboratory furnace to remove the residual chemicals and improve the quality of the IGZO film.

The crystal structure of the sample was characterized by $\mathrm{X}$-ray diffraction (XRD) using the copper $K_{\alpha 1}$ line under an accelerating voltage of $40 \mathrm{kV}$. The thermal behavior of the IGZO precursor solution was studied by using a thermogravimetric analyzer, which is operated at temperature ranging from room temperature to $800{ }^{\circ} \mathrm{C}$. The thickness of the IGZO film was carried out by a surface profiler. The film morphology was characterized by atomic force microscopy (AFM). The
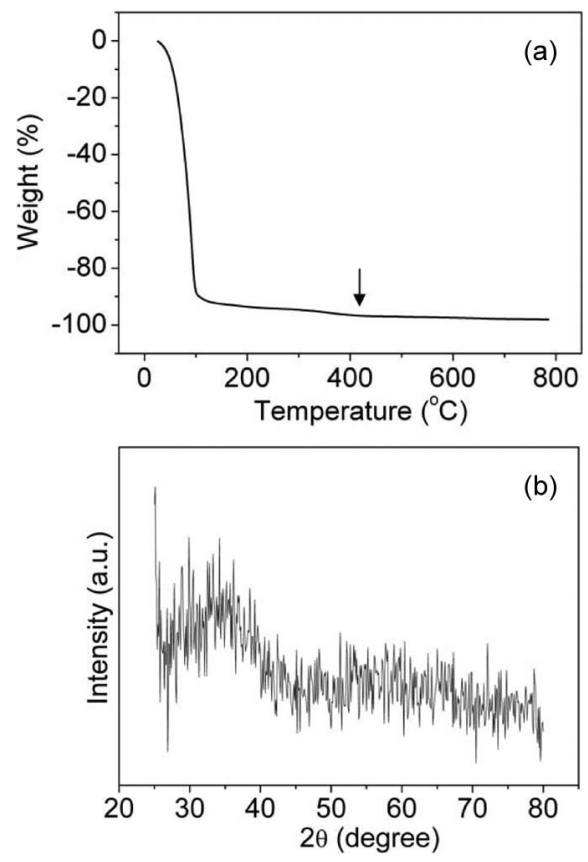

Fig. 2. (a) TGA curves of the IGZO ink. The arrow indicates the temperature that completes the conversion of the IGZO thin film. (b) XRD pattern of the IGZO thin film on a Si substrate annealed at $500{ }^{\circ} \mathrm{C}$ in the air for $1 \mathrm{~h}$.

transistor performance of the IGZO TFT was measured with an HP 4156A semiconductor parameter analyzer. The device fabrication and characterization were all conducted under ambient conditions without taking precautionary measures to avoid ambient lights, moisture, and oxygen.

\section{Results AND Discussions}

The IGZO inks dispensed from the inkjet head had a diameter of 6-8 $\mu \mathrm{m}$ and a volume of $1 \mathrm{pl}$. To get a better printed pattern, the ink flying speed between the nozzle and the substrate was precisely controlled by adjusting the firing voltage. The printed IGZO dots and single line are shown in Fig. 1(a) and (b), respectively. The dots were printed with a spacing of $100 \mu \mathrm{m}$ (between the centers of any two adjacent drops). A donut ring shape formed by a single drop can be seen in the inset of Fig. 1(a). The line was made up of printed dots with $5-\mu \mathrm{m}$ drop spacing. We can see that the printed IGZO film is uniform at the center. The thickness of the film can be easily adjusted by controlling the inkjetting frequency and the printing times. Typical optical images of printed IGZO TFTs with bottomgate bottom-contact device architectures are shown in Fig. 1(c). As we can see, the IGZO ink was only dropped between the source-drain electrodes without wasting ink, i.e., a drop-ondemand process [22].

Thermogravimetric analysis (TGA) was performed in atmosphere with a heating rate of $5{ }^{\circ} \mathrm{C} / \mathrm{min}$ to determine the postannealing temperature for IGZO thin films. As shown in Fig. 2(a), the conversion of the oxide film was completed around $420{ }^{\circ} \mathrm{C}$. Most weight loss below $420^{\circ} \mathrm{C}$ was attributed to the evaporation of the solvent, decomposition, hydrolysis, and dehydroxylation from the precursors [10], [18]. Therefore, a $500-{ }^{\circ} \mathrm{C}$ annealing temperature is high enough for the 

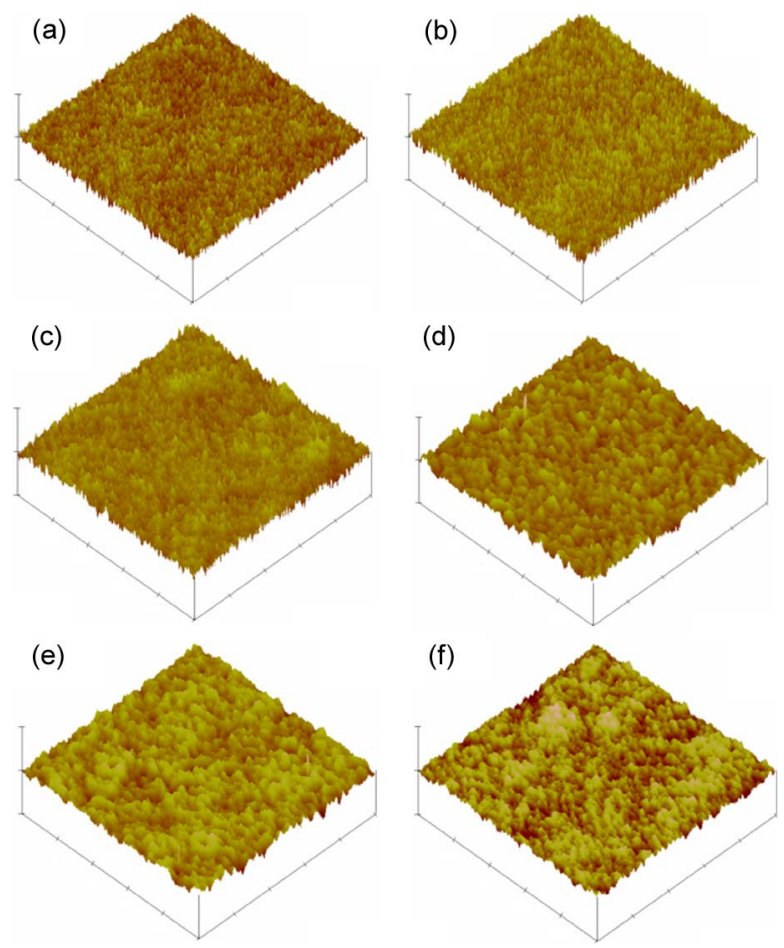

Fig. 3. AFM images of surface morphology of the inkjet-printed IGZO film with the thickness of (a) $23 \mathrm{~nm}$, (b) $41 \mathrm{~nm}$, (c) $55 \mathrm{~nm}$, (d) $78 \mathrm{~nm}$, (e) $103 \mathrm{~nm}$, and (f) $125 \mathrm{~nm}$. The size of all images is $1 \mu \mathrm{m} \times 1 \mu \mathrm{m}$.

TABLE I

RMS Roughness of the IGZO FILMS With DifFERENT THICKNESSES

\begin{tabular}{cc}
\hline \hline Thickness $(\mathrm{nm})$ & RMS roughness $(\mathrm{nm})$ \\
\hline 23 & 0.189 \\
41 & 0.270 \\
55 & 0.320 \\
78 & 0.333 \\
103 & 0.322 \\
125 & 0.337 \\
\hline \hline
\end{tabular}

formation of IGZO thin films. Fig. 2(b) shows the XRD pattern of IGZO films. We can see that the film is amorphous. It was known that the amorphous film has the advantage of large area uniformity, which is crucial for production. Moreover, amorphous films have other added advantages, including smooth surface, and low interface state density and low electronic-defect domains [23].

The morphology of the IGZO thin films with different thicknesses is compared in Fig. 3(a)-(f), and their root mean square (RMS) roughness is summarized in Table I. It can be seen that, with the increase in IGZO thickness, the RMS roughness monotonically increases from 0.189 to $0.33 \mathrm{~nm}$ for a film thickness of 23, 41, and $55 \mathrm{~nm}$. The film was deposited by repeating a few cycles of printing and annealing to obtain the desired film thickness (the thickness is controlled by the number of cycles); therefore, the RMS roughness increases with the increase in thickness. For films thicker than $55 \mathrm{~nm}$, the RMS roughness does not significantly change. The largest RMS roughness of our inkjet-printed IGZO film is only $0.337 \mathrm{~nm}$. Thus, the inkjet-printed IGZO surface is very smooth and uniform due to the amorphous nature of the film, which is suitable for TFT application.
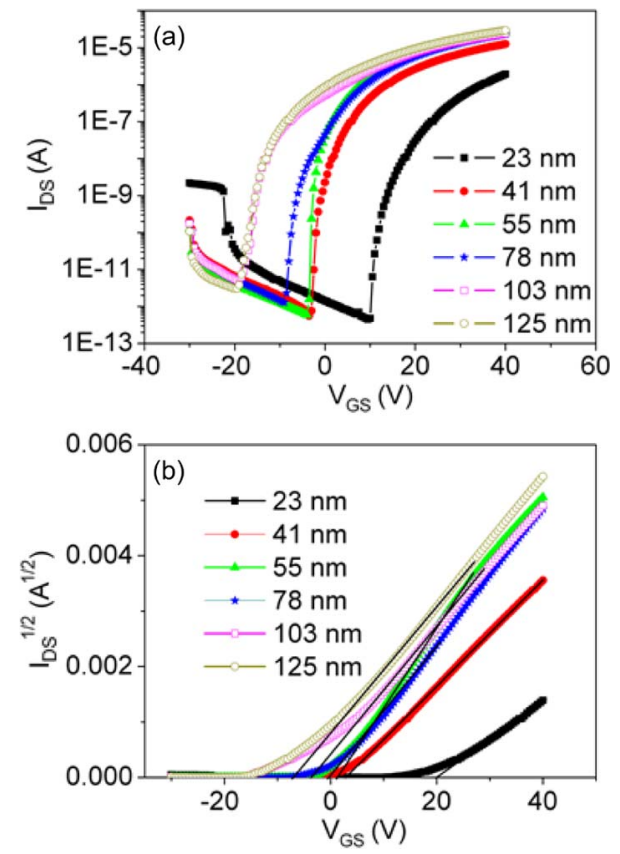

Fig. 4. Device performance for inkjet-printed TFTs with different IGZO film thicknesses. (a) Transfer characteristics and (b) the square root of $I_{D S}-V_{G S}$ at $V_{D S}=20 \mathrm{~V}$ for TFTs with different IGZO film thicknesses.

Using the inkjet-printed IGZO film annealed at $500{ }^{\circ} \mathrm{C}$ in the air, we fabricated bottom-gate bottom-contact structured IGZO TFTs with $W / L=200 \mu \mathrm{m} / 200 \mu \mathrm{m}$ and without passivation, and their device performance is shown in Fig. 4. Fig. 4(a) summarizes the transfer characteristics at $V_{D S}=20 \mathrm{~V}$ for TFTs with different IGZO film thicknesses. Fig. 4(b) is the square root of $I_{D S}-V_{G S}$ to show the threshold voltage and the field-effect mobility in the saturation region. Fig. 5(a)-(f) presents their output characteristics. It can be seen that the thickness of the channel layer has a strong impact on the TFT performance [24]. The best performance was obtained for the TFT with 55-nm-thick IGZO thin film. It was operated in n-channel enhancement mode with a field-effect mobility in the saturation region of $1.41 \mathrm{~cm}^{2} / \mathrm{V} \cdot \mathrm{s}$, a threshold voltage of $1 \mathrm{~V}$, a drain current on/off ratio of approximately $4.3 \times 10^{7}$, a subthreshold swing of $384 \mathrm{mV} / \mathrm{dec}$, and an off-current level of lower than $1 \mathrm{pA}$. Compared to the device performance of the previously reported inkjet-printed IGZO TFTs (with a field-effect mobility of $0.03 \mathrm{~cm}^{2} / \mathrm{V} \cdot \mathrm{s}$ and on/off-current ratio $\sim 10^{4}$ ) [21], our result is significantly improved. The mobility of inkjet-printed IGZO TFT $\left(1.41 \mathrm{~cm}^{2} / \mathrm{V} \cdot \mathrm{s}\right)$ is also slightly better than that of the spin-coating one $\left(0.96 \mathrm{~cm}^{2} / \mathrm{V} \cdot \mathrm{s}\right)$ [18].

The device performance of IGZO TFTs with different thicknesses of channel layer is summarized in Table II. A general observation is that, with the increase in the thickness of the IGZO film, the field-effect mobility is first increased and then slightly decreased, threshold voltage is shifted from positive to negative, off-drain current is increased, on-drain current is first increased and then saturated, and subthreshold swing is fluctuated in a small range for thin films and then increased for the thicker ones; these observed phenomena are in agreement with the case of ZnO TFTs [25]-[28]. Lower carrier mobility was commonly obtained for thinner IGZO films. The reason is 

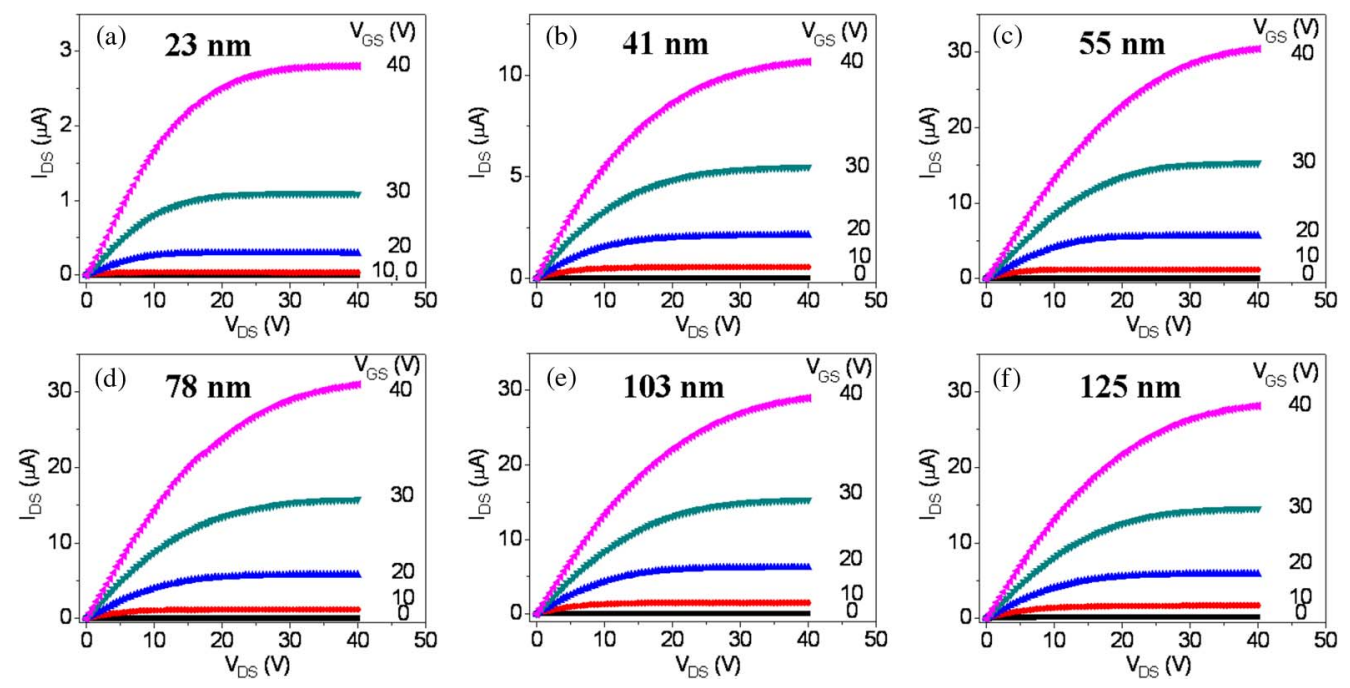

Fig. 5. Output characteristics for inkjet-printed TFTs with different IGZO film thicknesses of (a) $23 \mathrm{~nm}$, (b) $41 \mathrm{~nm}$, (c) $55 \mathrm{~nm}$, (d) $78 \mathrm{~nm}$, (e) $103 \mathrm{~nm}$, and (f) $125 \mathrm{~nm}$.

mainly because, as the carrier transport layer is farther from the surface of the thicker film [29], the influence of surface roughness on the carrier mobility is weaker in the thicker film, compared with the thinner film. Therefore, higher mobility was obtained in the thicker film, as we can see from the Table II. However, with the increase in film thickness thicker than $55 \mathrm{~nm}$, the field-effect mobility decreased from 1.41 to $1.16 \mathrm{~cm}^{2} / \mathrm{V} \cdot \mathrm{s}$. The reason is due to an increased carrier scattering associated with increased trap density as thickness is increased. The same trend was also found in solution-processed $\mathrm{ZnO}$ [25]. It is generally accepted that, for a given TFT geometry, it is possible to define an optimum semiconductor film thickness ensuring maximum TFT performance [24]. For example, the highest mobility for the a-Si:H TFT with a channel length of $100 \mu \mathrm{m}$ was obtained by a 100-150-nm-thick a-Si:H semiconductor layer [24]. In our device, the highest mobility device is obtained by $50-80$-nm-thick IGZO film. The operation mode changes from enhancement mode (positive threshold voltage) to depletion mode (negative threshold voltage) with the increase in the thickness of the IGZO film. In order to explain this phenomenon, we need to consider the influence of the oxygen on the surface of the IGZO film [21], [30]. As well known, surface-absorbed oxygen attracts electrons in IGZO films and forms a depletion layer below the surface [31], [32]. The width of the depletion layer can be estimated by

$$
W=\left(\frac{2 \varepsilon_{I G Z O} \varphi_{s}}{e N_{D}}\right)^{1 / 2}
$$

where $\varphi_{s}$ is the surface barrier potential, $e$ is the electronic charge, $N_{D}$ is the doping concentration, and $\varepsilon_{\mathrm{IGZO}}$ is the dielectric constant of IGZO [33]. By assuming similar parameters to $\mathrm{ZnO}$, i.e., $N_{D}=10^{17} / \mathrm{cm}^{3}$, $\varepsilon_{\mathrm{IGZO}}=8.66$, and $\varphi_{s}=0.3 \mathrm{eV}$, we obtain the width of the depletion layer to be $W=54 \mathrm{~nm}$ [33]. When the film thickness is less than $W$, the threshold voltage is positive because the thinner IGZO TFT is completely depleted under zero gate bias. While the thickness is larger than $W$, the threshold voltage is negative because the TFT is partially depleted under zero gate bias. This result indicates that the surface depletion has a strong impact on the performance of the IGZO TFTs for ultrathin films. In addition, it also indicates that the threshold voltage can be adjusted to zero when the film thickness is in the range of $50-80 \mathrm{~nm}$, which is in good agreement with our result $(1$ and $-0.5 \mathrm{~V}$ for $55-$ and $78-\mathrm{nm}$ IGZO films, respectively). This explains why the inkjet-printed IGZO TFT works in enhancement mode when the thickness is less than $50 \mathrm{~nm}$, whereas it works in depletion mode when the film thickness is thicker than $80 \mathrm{~nm}$. Due to the higher resistivity of the thinner film, the off current is smaller than that of the thicker film.

With the increase in film thickness, the subthreshold swing fluctuates within a small range for IGZO films thinner than $55 \mathrm{~nm}$ and then increases for the film thicker than $55 \mathrm{~nm}$. The turning point of $55 \mathrm{~nm}$ coincides with the depletion layer thickness obtained from (1). For films thinner than $55 \mathrm{~nm}$, the channel is fully depleted, leading to a small subthreshold swing. It is indeed the film thickness that determines the subthreshold swing change.

From the value of the subthreshold swing, the sheet trap density $N_{T}$ (with a unit of $\mathrm{cm}^{-2}$ ) can be estimated by

$$
N_{T}=\left(\frac{S\left(\log _{10} e\right)}{k T / q}-1\right) \frac{C_{i}}{q}
$$

where $S$ is the subthreshold swing, $k$ is the Boltzmann's constant, $T$ is the temperature, and $C_{i}$ is the unit gate capacitance [34], [35]. From the equation, we can see that the subthreshold swing is in proportion to the trap density. The degradation of the subthreshold swing with the increase in the film for the thicker film devices is due to a combination of effects: an increase in off drain current and increase in sheet trap density $N_{T}$ [36]. A detailed mechanism of the degradation of subthreshold swing induced by the increased off drain current can be found from [36]. With the increase in semiconductor thickness, the distance between the charge centroid and $\mathrm{IGZO} / \mathrm{SiO}_{2}$ interface increases; then, a semiconductor capacitance is inserted in series with the gate insulator capacitance, reducing the effective capacitance and leading to an increase in subthreshold 
TABLE II

Comparison of the Various Parameters for the InK-Jet Printed TFTs With Different IGZO Film Thicknesses

\begin{tabular}{|c|c|c|c|c|c|}
\hline $\begin{array}{l}\text { Thickness } \\
\text { (nm) }\end{array}$ & $\begin{array}{l}\text { Saturation mobility } \\
\left(\mathrm{cm}^{2} / \mathrm{V} \mathrm{s}\right)\end{array}$ & $\begin{array}{c}\text { Threshold } \\
\text { voltage (V) }\end{array}$ & $\mathrm{I}_{\mathrm{on}} / \mathrm{I}_{\mathrm{off}}$ & $\begin{array}{l}\text { Subthreshold swing } \\
\text { (V/dec) }\end{array}$ & $\begin{array}{l}\text { Trap density } \\
\left(10^{12} / \mathrm{cm}^{2}\right)\end{array}$ \\
\hline 23 & 0.40 & 20 & $4.0 \mathrm{E} 6$ & 0.434 & 0.901 \\
\hline 41 & 0.82 & 3.5 & $2.4 \mathrm{E} 7$ & 0.451 & 0.943 \\
\hline 55 & 1.41 & 1.0 & $4.3 \mathrm{E} 7$ & 0.384 & 0.781 \\
\hline 78 & 1.36 & -0.5 & $1.8 \mathrm{E} 7$ & 0.584 & 1.260 \\
\hline 103 & 1.16 & -3.5 & 4.6E6 & 0.909 & 2.189 \\
\hline 125 & 1.19 & -6.5 & $9.6 \mathrm{E} 6$ & 1.38 & 3.181 \\
\hline
\end{tabular}

swing [36]. Subsequently, we consider the increase in sheet trap density. Suppose that the channel layer trap density $n_{t}$ is constant across the entire film. Then, $N_{T}$ is proportional to IGZO film thickness, which leads to an increase in $N_{T}$ for a thicker film [36]. More traps induce more free carriers, resulting in the subthreshold swing degradation in depletion-mode TFTs, which has been observed in ZTO TFTs [37].

\section{CONCLUSION}

In conclusion, we have fabricated IGZO TFTs with bottomgate bottom-contact device architectures by inkjet printing method. The influence of the IGZO film thickness (23-125 nm) on the performance of TFTs has been discussed in detail. The threshold voltage, mobility, on/off drain current, and subthreshold swing can be modified by varying IGZO film thickness. The printed IGZO TFTs with an optimal 55-nm thickness annealed at $500{ }^{\circ} \mathrm{C}$ in the air showed a field-effect mobility in the saturation region of $1.41 \mathrm{~cm}^{2} / \mathrm{V} \cdot \mathrm{s}$, a threshold voltage of $1 \mathrm{~V}$, a drain current on/off ratio of approximately $4.3 \times 10^{7}$, a subthreshold swing of $384 \mathrm{mV} / \mathrm{dec}$, and an off-current level lower than $1 \mathrm{pA}$. The performance of the inkjet-printed IGZO can be compared with that of spin-coated IGZO TFTs. Our results demonstrate the possibility of fabricating IGZO TFTs by inkjet printing technology, which is amenable to roll-to-roll manufacturing process in the ambient condition with low cost.

\section{REFERENCES}

[1] Y. L. Wu, Y. N. Li, and B. S. Ong, "A simple and efficient approach to a printable silver conductor for printed electronics," J. Amer. Chem. Soc., vol. 129, no. 7, pp. 1862-1863, Feb. 2007.

[2] B. J. de Gans, P. C. Duineveld, and U. S. Schubert, "Inkjet printing of polymers: State of the art and future developments," Adv. Mater., vol. 16, no. 3, pp. 203-213, Feb. 2004.

[3] B. Ong, "Semiconductor ink advances flexible displays," Laser Focus World, vol. 40, no. 6, pp. 85-88, Jun. 2004.

[4] T. Shimoda, Y. Matsuki, M. Furusawa, T. Aoki, I. Yudasaka, H. Tanaka, H. Iwasawa, D. H. Wang, M. Miyasaka, and Y. Takeuchi, "Solutionprocessed silicon films and transistors," Nature, vol. 440, no. 7085, pp. 783-786, Apr. 2006.

[5] H. Yan, Z. H. Chen, Y. Zheng, C. Newman, J. R. Quinn, F. Dötz, M. Kastler, and A. Facchetti, "A high-mobility electron-transporting polymer for printed transistors," Nature, vol. 457, no. 7230, pp. 679-686, Feb. 2009

[6] M. Berggren, D. Nilsson, and N. D. Robinson, "Organic materials for printed electronics," Nat. Mater, vol. 6, no. 1, pp. 3-5, Jan. 2007.

[7] J. Z. Wang, Z. H. Zheng, H. W. Li, W. T. S. Huck, and H. Sirringhaus, "Dewetting of conducting polymer inkjet droplets on patterned surfaces," Nat. Mater., vol. 3, no. 3, pp. 171-176, Mar. 2004.

[8] D. H. Lee, Y. J. Chang, G. S. Herman, and C. H. Chang, "A general route to printable high-mobility transparent amorphous oxide semiconductors," Adv. Mater., vol. 19, no. 6, pp. 843-847, Mar. 2007.
[9] S. Y. Han, D. H. Lee, G. S. Herman, and C. H. Chang, "Inkjet-printed high mobility transparent-oxide semiconductors," J. Display Technol., vol. 5, no. 12 , pp. 520-524, Dec. 2009.

[10] D. H. Lee, S. Y. Han, G. S. Herman, and C. H. Chang, "Inkjet printed high-mobility indium zinc tin oxide thin film transistors," J. Mater. Chem., vol. 19, no. 20, pp. 3135-3137, 2009.

[11] K. Nomura, H. Ohta, A. Takagi, T. Kamiya, M. Hirano, and H. Hosono, "Room-temperature fabrication of transparent flexible thin-film transistors using amorphous oxide semiconductors," Nature, vol. 432, no. 7016, pp. 488-492, Nov. 2004.

[12] K. Nomura, H. Ohta, K. Ueda, T. Kamiya, M. Hirano, and H. Hosono, "Thin-film transistor fabricated in single-crystalline transparent oxide semiconductor," Science, vol. 300, no. 5623, pp. 1269-1272, May 2003.

[13] J. K. Jeong, J. H. Jeong, H. W. Yang, J. S. Park, Y. G. Mo, and H. D. Kim, "High performance thin film transistors with cosputtered amorphous indium gallium zinc oxide channel," Appl. Phys. Lett., vol. 91, no. 11, p. 113505 , Sep. 2007.

[14] H. Yabuta, M. Sano, K. Abe, T. Aiba, T. Den, H. Kumomi, K. Nomura, T. Kamiya, and H. Hosono, "High-mobility thin-film transistor with amorphous $\mathrm{InGaZnO}_{4}$ channel fabricated by room temperature rf-magnetron sputtering," Appl. Phys. Lett., vol. 89, no. 11, p. 112 123, Sep. 2006.

[15] M. Kim, J. H. Jeong, H. J. Lee, T. K. Ahn, H. S. Shin, J. S. Park, J. K. Jeong, Y. G. Mo, and H. D. Kim, "High mobility bottom gate InGaZnO thin film transistors with $\mathrm{SiO}_{x}$ etch stopper," Appl. Phys. Lett., vol. 90, no. 21, p. 212114 , May 2007.

[16] J. Park, C. Kim, S. Kim, H. Song, D. Kang, H. Lim, H. Yin, R. Jung, E. Lee, J. Lee, K. W. Kwon, and Y. Park, "Source/drain series-resistance effects in amorphous gallium-indium zinc-oxide thin film transistors," IEEE Electron Device Lett., vol. 29, no. 8, pp. 879-881, Aug. 2008.

[17] H. Q. Chiang, B. R. McFarlane, D. Hong, R. E. Presley, and J. F. Wager, "Processing effects on the stability of amorphous indium gallium zinc oxide thin-film transistors," J. Non-Cryst. Solids, vol. 354, no. 19-25, pp. 2826-2830, May 2008

[18] G. H. Kim, H. S. Shin, B. D. Ahn, K. H. Kim, W. J. Park, and H. J. Kim, "Formation mechanism of solution-processed nanocrystalline InGaZnO thin film as active channel layer in thin-film transistor," J. Electrochem. Soc., vol. 156, no. 1, pp. H7-H9, 2009.

[19] J. H. Lim, J. H. Shim, J. H. Choi, J. Joo, K. Park, H. Jeon, M. R. Moon, D. Jung, H. Kim, and H. J. Lee, "Solution-processed InGaZnO-based thin film transistors for printed electronics applications," Appl. Phys. Lett., vol. 95, no. 1, p. 012 108, Jul. 2009.

[20] Y. Wang, S. W. Liu, X. W. Sun, J. L. Zhao, G. K. L. Goh, Q. V. Vu, and H. Y. Yu, "Highly transparent solution processed In-Ga-Zn oxide thin films and thin film transistors," J. Sol-Gel Sci. Technol., vol. 55, no. 3, pp. 322-327, Sep. 2010.

[21] G. H. Kim, H. S. Kim, H. S. Shin, B. D. Ahn, K. H. Kim, and H. J. Kim, "Inkjet-printed InGaZnO thin film transistor," Thin Solid Films, vol. 517, no. 14, pp. 4007-4010, May 2009.

[22] H. Sirringhaus and T. Shimoda, "Inkjet printing of functional materials," MRS Bull., vol. 28, no. 11, pp. 802-803, Nov. 2003.

[23] J. T. Anderson, C. L. Munsee, C. M. Hung, T. M. Phung, G. S. Herman, D. C. Johnson, J. F. Wager, and D. A. Keszler, "Solution-processed HafSOx and ZircSOx inorganic thin-film dielectrics and nanolaminates," $A d v$. Funct. Mater., vol. 17, no. 13, pp. 2117-2124, Sep. 2007.

[24] S. Martin, C. S. Chiang, J. Y. Nahm, T. Li, J. Kanicki, and Y. Ugai, "Influence of the amorphous silicon thickness on top gate thin-film transistor electrical performances," Jpn. J. Appl. Phys., vol. 40, no. 2A, pp. 530537, Feb. 2001.

[25] C. S. Li, Y. N. Li, Y. L. Wu, B. S. Ong, and R. O. Loutfy, "Fabrication conditions for solution-processed high-mobility $\mathrm{ZnO}$ thin-film transistors," $J$. Mater. Chem., vol. 19, no. 11, pp. 1626-1634, 2009. 
[26] D. Redinger and V. Subramanian, "High-performance chemical-bathdeposited zinc oxide thin-film transistors," IEEE Trans. Electron Devices, vol. 54, no. 6, pp. 1301-1307, Jun. 2007.

[27] J. H. Chung, J. Y. Lee, H. S. Kim, N. W. Jang, and J. H. Kim, "Effect of thickness of $\mathrm{ZnO}$ active layer on $\mathrm{ZnO}$-TFTs characteristics," Thin Solid Films, vol. 516, no. 16, pp. 5597-5601, Jun. 2008.

[28] B. Y. Oh, M. C. Jeong, M. H. Ham, and J. M. Myoung, "Effects of the channel thickness on the structural and electrical characteristics of room-temperature fabricated $\mathrm{ZnO}$ thin-film transistors," Semicond. Sci. Technol., vol. 22, no. 6, pp. 608-612, Jun. 2007.

[29] R. L. Hoffman, B. J. Norris, and J. F. Wager, "ZnO-based transparent thinfilm transistors," Appl. Phys. Lett., vol. 82, no. 5, pp. 733-735, Feb. 2003.

[30] S. Jeong, Y. G. Ha, J. Moon, A. Facchetti, and T. J. Marks, "Role of gallium doping in dramatically lowering amorphous-oxide processing temperatures for solution-derived indium zinc oxide thin-film transistors," Adv. Mater, vol. 22, no. 12, pp. 1346-1350, Mar. 2010.

[31] J. S. Park, J. K. Jeong, H. J. Chung, Y. G. Mo, and H. D. Kim, "Electronic transport properties of amorphous indium-gallium-zinc oxide semiconductor upon exposure to water," Appl. Phys. Lett., vol. 92, no. 7, p. 072 104, Feb. 2008.

[32] J. K. Jeong, H. W. Yang, J. H. Jeong, Y. G. Mo, and H. D. Kim, “Origin of threshold voltage instability in indium-gallium-zinc oxide thin film transistors," Appl. Phys. Lett., vol. 93, no. 12, p. 123 508, Sep. 2008.

[33] W. K. Hong, J. I. Sohn, D. K. Hwang, S. S. Kwon, G. Jo, S. Song, S. M. Kim, H. J. Ko, S. J. Park, M. E. Weiland, and T. Lee, "Tunable electronic transport characteristics of surface-architecture-controlled $\mathrm{ZnO}$ nanowire field effect transistors," Nano Lett., vol. 8, no. 3, pp. 950-956, Mar. 2008.

[34] C. R. Kagan and P. Andry, Thin-Film Transistors, 1st ed. New York: Marcel Dekker, 2003.

[35] D. W. Greve, Field Effect Devices and Applications: Devices for Portable, Low-Power, and Imaging Systems., 1st ed. Englewood Cliffs, NJ: Prentice-Hall, 1998, pp. 287-288.

[36] H. Q. Chiang, "Development of oxide semiconductors: Materials, devices, and integration," Ph.D. dissertation, Dept. Elect. Comput. Eng., Oregon State Univ., Corvallis, OR, 2007.

[37] W. B. Jackson, R. L. Hoffman, and G. S. Herman, "High-performance flexible zinc tin oxide field-effect transistors," Appl. Phys. Lett., vol. 87, no. 19 , p. 193503 , Nov. 2005

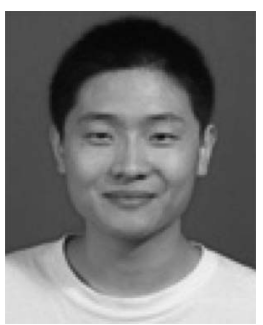

Ye Wang was born in China in 1981. He received the B.Sc. degree in physics from Zhengzhou University, Zhengzhou, China, in 2004 and the M.Sc. degree in condensed matter physics from the University of Science and Technology of China, Hefei, China, in 2007. He is currently working toward the Ph.D. degree with the Nanoelectronic Group, Division of Microelectronics, School of Electrical and Electronic Engineering, Nanyang Technological University, Singapore.

His research interests include inkjet-printed In-Ga-Zn oxide thin-film transistors and $\mathrm{ZnO}$-related inorganic materials, including film and nanowires.

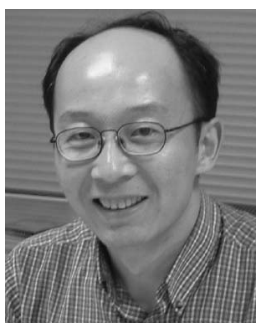

Xiao Wei Sun (SM'06) was born in Beijing, China, in 1968. He received the B.Eng., M.Eng., and Ph.D. degrees in photonics from Tianjin University, Tianjin, China, in 1990, 1992, and 1996, respectively, and the Ph.D. degree in electrical and electronic engineering from Hong Kong University of Science and Technology, Kowloon, Hong Kong, in 1998.

In 1998, he joined the Division of Microelectronics, School of Electrical and Electronic Engineering, Nanyang Technological University, Singapore, as an Assistant Professor, and was promoted to Associate Professor in October 2005. $\mathrm{He}$ is currently the Dean of the College of Science, Tianjin University. He has been a Guest Professor of Southeast University, Nanjing, China. He has (co)authored more than 250 peer-reviewed journal publications in the area of photonics and microelectronics.

Dr. Sun is a Fellow of the Institute of Physics (FInstP) and a member of Society for Information Display. He is the Founder and Director of SID Singapore and Malaysia Chapter. He is the recipient of the Nanyang Award for Research and Innovation 2009 for his contribution in $\mathrm{ZnO}$ nanodevices.

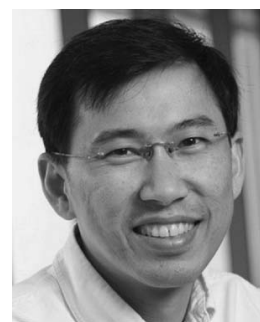

Gregory Kia Liang Goh received the M.Eng. and B.Eng. degrees from the National University of Singapore, Singapore, and the Ph.D. degree from the University of California, Santa Barbara.

$\mathrm{He}$ is currently a Research Scientist with the Institute of Materials Research and Engineering (IMRE), Agency for Science, Technology and Research (A*STAR), Singapore, and was previously the Head of the Materials Growth Capability Group from 2007 to 2009. At IMRE, he leads a team utilizing low-temperature solutions to growing oxide films and nanostructures for light-emitting diode, ferroelectric, photovoltaic, and photocatalytic applications. His work has been published in a book by American Scientific Publishers that summarizes the growth and integration of nanostructured materials by solution methods and the effect of the growth solution on subsequent properties.

Dr. Goh is an Associate Editor for Nanoscience and Nanotechnology Letters (USA) and a Technical Advisor for Advanced Materials Technologies Pte Ltd.

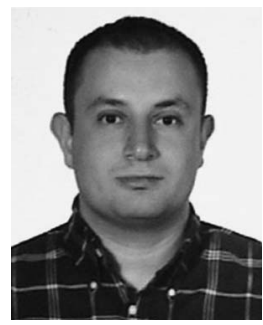

Hilmi Volkan Demir (S'97-M'04) received the B.Sc. degree in electrical and electronics engineering from Bilkent University, Ankara, Turkey, in 1998 and the M.S. and Ph.D. degrees in electrical engineering from Stanford University, Stanford, CA, in 2000 and 2004, respectively.

In 2004, he joined Bilkent University as a faculty member and is an Associate Professor with joint appointments at the Department of Electrical and Electronics Engineering and the Department of Physics. In 2007, he received the Docent title (associate professorship) from the Turkish Council of Higher Education. In 2009, he has been awarded Singapore NRF Fellowship. He is concurrently Nanyang Associate Professor jointly with the School of Electrical and Electronic Engineering (Microelectronics Division) and the School of Physical and Mathematical Sciences (Physics and Applied Physics Division), Nanyang Technological University, Singapore, and the Director of the Luminous! Center of Excellence for Semiconductor Lighting and Displays. His current research interests include the development of innovative devices and sensors, including the science and technology of excitonics for high-efficiency light generation and harvesting and wireless implant sensing for future healthcare.

Dr. Demir was the recipient of the European Science Foundation European Young Investigator Award in 2007 and the National Scientific Technological Research Council Distinguished Young Scientist Award of Turkey in 2009.

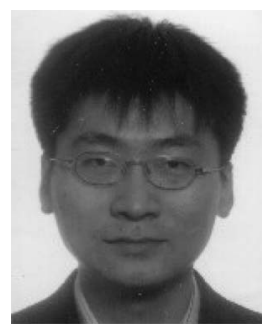

Hong Yu Yu (SM'10) received the B.Eng. degree from Tsinghua University, Beijing, China, in 1999, the M.A.Sc. degree from Toronto University, Toronto, ON, Canada, in 2001, and the Ph.D. degree from the National University of Singapore (NUS), Singapore, in 2005.

In January 25, 2008, he joined the School of Electrical and Electronic Engineering of Nanyang Technological University in Singapore. From June 2004 to January 2008, he was a Senior Researcher in the area of Si technologies within IMEC, Belgium. He has authored or coauthored more than 180 publications in top-tied international journals and conferences (including one highlight paper in VLSI-07) and three book chapters. He is the holder of more than 20 USA/EU patents. His research interests include emerging Si-based nanoelectronic device for both "More Moore" and "More than Moore" applications, e.g., novel nonvolatile memories, sub-22-nm CMOS devices, advanced and photovoltaic devices.

Dr. Yu was awarded with a NUS president graduate fellowship and an IEEE EDS graduate fellowship during his Ph.D. candidature. 